\title{
TURISMO INCLUSIVO PARA TODAS LAS PERSONAS. UNA APUESTA POR LA DIVERSIDAD.
}

\author{
$M^{a}$ Carmen Martín Cano \\ Universidad de Jaén (España) \\ Encarnación Luque Serrano \\ A.S.C. Alternativas (España) \\ Yolanda $M^{\text {a }}$ de la Fuente Robles \\ Universidad de Jaén (España)
}

\begin{abstract}
Resumen. El turismo y el ocio son espacios fundamentales en la vida de cualquier persona, y por ende un derecho universal reconocido, como así queda reflejado en la Ley 51/2003, de Igualdad de Oportunidades, No Discriminación y Accesibilidad Universal de las Personas con Discapacidad. En este sentido, uno de los aspectos a trabajar en una sociedad avanzada es la accesibilidad al ocio y a la cultura para todas las personas. Cada vez son más las personas con discapacidad o diversidad funcional que demandan la posibilidad de ocupar su sitio en la actividad turística, y a las cuales se les debe garantizar este derecho. Por ello, en este trabajo lo que se pretende es analizar la situación actual y las posibles líneas de intervención en esta materia, concretamente en el turismo inclusivo para las personas con discapacidad o dificultad intelectual. A pesar de los avances que se están consiguiendo, no podemos obviar que este colectivo encuentra aún muchos problemas para hacer efectivo el derecho de acceder a un turismo de calidad. Así mismo se analizan los beneficios que el turismo aporta a todas las personas en general y a las personas con diversidad funcional en particular y, en consecuencia, el porqué de la necesidad del "Turismo para Todas las Personas" o "Turismo Inclusivo".
\end{abstract}

Palabras clave: Turismo Inclusivo, Diversidad funcional, Discapacidad Intelectual, Inclusión social.

\section{INCLUSIVE TOURISM FOR ALL. A COMMITMENT TO DIVERSITY}

\begin{abstract}
Tourism and leisure are key areas in the life of anyone, and therefore a universal right recognized, as well reflected in the Law 51/2003 of Equal 0portunidades, Non-Discrimination and Universal Accessibility of Persons with Disabilities. In this regard, one aspect to work in an advanced society is the accessibility to leisure and culture for all people.More and more people with disabilities or functional diversity that demand the ability to take his place in tourism, and to which they must guarantee this right. Therefore, in this work this aims to analyze the current situation and possible lines of intervention in this area, particularly in the inclusive tourism for people with intellectual disability or difficulty. Despite the advances that are being achieved, we can not ignore that this group still many problems to realize the right of access to quality tourism. Also the benefits that tourism brings to all people in general and people with disabilities in particular, and, therefore, why the need for "Tourism for All" or "Inclusive Tourism" are analyzed.
\end{abstract}

Keywords: Inclusive Tourism, functional diversity, Intellectual Disability, Social Inclusion 


\title{
TURISMO INCLUSIVA PARA TODOS. UM COMPROMISSO COM A DIVERSIDADE
}

\begin{abstract}
Resumo. Turismo e lazer são áreas fundamentais na vida de qualquer pessoa e, portanto, um direito universal, reconhecido, bem refletida na Lei 51/2003 de 0portunidades Igualdade, não-discriminação e Acessibilidade Universal das Pessoas com Deficiência. A este respeito, um aspecto para trabalhar em uma sociedade avançada é a acessibilidade para lazer e cultura para todas as pessoas. Mais e mais pessoas com deficiência ou com diversidade funcional que exigem a capacidade de tomar o seu lugar no turismo, e para o qual devem garantir esse direito. Portanto, neste trabalho, que tem como objetivo analisar a situação e as possíveis linhas atuais de intervenção nesta área, particularmente no turismo inclusivo para as pessoas com deficiência intelectual ou dificuldade. Apesar dos avanços que estão sendo alcançados, não podemos ignorar que este grupo ainda muitos problemas para realizar o direito de acesso a um turismo de qualidade. Também os benefícios que o turismo traz para todas as pessoas em geral e as pessoas com deficiência, em particular, e, portanto, por que a necessidade de "Turismo para Todos" ou "Turismo Inclusivo" são analisados.
\end{abstract}

Palavras-chave: turismo inclusivo, diversidade funcional, deficiência intelectual, inclusão social.

\section{Introducción}

Las nociones de igualdad de oportunidades y diseño para todas las personas se configuran como conceptos fundamentales respecto a una visión global de accesibilidad social. Así, los valores de este nuevo paradigma guían la accesibilidad hacia una nueva cultura de satisfacción igualitaria de necesidades, dejando a un lado la tradicional concepción de discapacidad, asumiendo la diversidad como norma y no como excepción ya que todas las personas, en mayor o menor medida, en determinados momentos de su vida son susceptibles de tener limitaciones. Esto es, no solo puede ser para una persona con unas determinadas condiciones inaccesible un entorno, sino también una información o un recurso dando lugar a imposibilitar la participación social, lo que conlleva a una clara desigualdad en relación a las personas que no la sufren.

El artículo 9 de La Convención de los Derechos de las Personas con Discapacidad (2006) se establece que para que las personas con discapacidad puedan vivir de forma independiente y participar plenamente en todos los aspectos de la vida, los Estados Partes deben tomar las medidas apropiadas para garantizar a las personas con discapacidad el acceso, en igualdad de condiciones con los demás, al entorno físico, el transporte, la información y las comunicaciones, las tecnologías y sistemas, así como otras instalaciones y servicios públicos o abiertos al público (De la Fuente, Martín y Hernández, 2016).

Asimismo, el European Institute for Design and Disability señalaba la necesidad de tener en cuenta desde el principio las limitaciones y las capacidades de las personas, y así desarrollar bienes y servicios utilizables por toda la población, mejorando su calidad de vida a través del denominado design for all o diseño para todas las personas. (EIDD, 2004)

Obviamente, las nociones de Accesibilidad Universal y Diseño para Todas las personas van más allá del entorno físico y virtual presentando un marcado aspecto social. Así, desde Europa se entiende y define el término accesibilidad con tres formas 
básicas de actividad humana: la movilidad, la comunicación y la comprensión. Del mismo modo, y sin dejar el ámbito europeo, la Declaración de Estocolmo del Diseño para Todos resumía sus reivindicaciones bajo la afirmación "el buen diseño capacita, el mal diseño discapacita".

Por ello, el reto de la sociedad actual es que todas las personas tengan las mismas posibilidades de desarrollar una vida digna y decidir sobre su actividad, vivienda o estilo de vida. Desde esta perspectiva, el eje central del diseño para todas las personas se sitúa en la búsqueda de soluciones de diseño para que todas las personas, independientemente de la edad, el género, las capacidades físicas, psíquicas y sensoriales o su bagaje cultural, puedan utilizar los espacios, productos y servicios de su entorno y, al mismo tiempo, participar en la construcción de nuestra sociedad.

En este sentido, uno de los aspectos a trabajar en una sociedad avanzada es la accesibilidad al ocio y a la cultura para todos/as los/as ciudadanos/as. Cada vez son más las personas con discapacidad o diversidad funcional que demandan la posibilidad de ocupar su sitio en la actividad turística, y se debe garantizar su derecho a cubrir estas necesidades.

Las sociedades incluyentes deben de garantizar el derecho al turismo de todas las personas. Se debe trabajar por tanto en pro de la consecución de un turismo inclusivo, dando un paso más allá del turismo accesible, un turismo para todas las personas cuyo uso universal nos garantice la existencia de un turismo de calidad.

Por otro lado, centrándonos en las necesidades de las personas con discapacidad intelectual podemos destacar que presentan dificultades para comprender las situaciones emergentes a su alrededor, teniendo en un gran número de ocasiones una visualización alterna de la realidad, esto es minimizando o exacerbando el hecho, por lo que la información adecuada, de fácil lectura y comprensión, se constituyen como una herramienta prioritaria para su actuar en determinadas situaciones. (Garza, 2016)

Por ello, el presente artículo tiene como objetivo fundamental realizar un análisis sobre el porqué del turismo accesible e inclusivo en la sociedad actual como recurso emergente ante las nuevas realidades y nuevos retos que presenta el siglo XXI de modo general, y de manera específica centrar nuestra atención en las necesidades y demandas que el colectivo de personas con discapacidad intelectual presenta en la materia en cuestión. Igualmente analizar cuáles son las herramientas y dispositivos que debemos poner en marcha para la consecución de un Turismo para todas las personas.

\section{Método}

De acuerdo con los objetivos planteados, el presente trabajo se constituye en una investigación bibliográfica de abordaje cualitativo. Para ello se ha llevado a cabo una revisión sistemática de literatura científica relacionada con nuestro objeto de estudio, consultando, entre otras, las bases de datos SCORPUS, SCIELO, DIALNET y diversas fuentes institucionales tales como la OMS y el SAAD

La revisión bibliográfica se configura como un estudio detallado, selectivo y crítico que integra la información esencial en una perspectiva unitaria y de conjunto (Iscart y Canela, 1994). En sí la revisión tiene como finalidad examinar la bibliografía publicada y situarla en cierta perspectiva (Ramos, Ramos y Romero (2003). Cabe destacar que la búsqueda bibliográfica en el contexto de la revisión se comprende como 
un estudio en sí mismo, en el cual quien la realiza tiene una incógnita, recoge datos, los analiza y extrae de ellos unas conclusiones. Como bien señala Guirao (2015), entre otras, tienen una función importante en la evaluación de las tendencias actuales así como en la formulación de propuestas de mejora.

\section{Resultados}

\section{Siglo XXI. Nuevo paradigma en Turismo.}

El turismo se ha convertido en motor clave de crecimiento socioeconómico y uno de los sectores que más están creciendo en la actualidad. Como prueba de ello debemos de citar dos fechas claves en la evolución del mismo:

- Año 2012. Se produce un hito histórico: Se sitúa en mil millones el número de personas que viajan por el mundo.

- Año 2013. Se establece el record mundial total de llegadas por todo el mundo con un total de mil ochenta y siete llegadas.

Todo ello nos ofrece un gran número de nuevas tendencias y a su vez nos plantea una serie de retos entre los cuales debemos destacar la oportunidad para promover la igualdad de género, la sostenibilidad ambiental, el comercio, el crecimiento económico y la promoción de la autonomía personal (Muñoz, 2015). Es prioritario, por tanto, trabajar en este ámbito para intentar dar respuesta a estos nuevos retos y a la demanda social generalizada que se está generando entorno al sector del turismo.

Entre estas nuevas demandas, debemos de destacar el papel fundamental del turismo accesible. Las personas con discapacidad o diversidad funcional, participan cada vez con mayor frecuencia en actividades turísticas como consecuencia de su creciente grado de integración económica y social. Sin embargo, aún persisten muchos impedimentos y barreras de todo tipo que dificultan el acceso a los servicios turísticos. La realidad demuestra que aún son muchas las personas con algún tipo de discapacidad que no viajan, a pesar de disponer tiempo libre y deseos de hacerlo, por temor a no encontrar destinos turísticos accesibles (Manual sobre Turismo accesible para todos, Principios, herramientas y buenas prácticas. Módulo I: Turismo Accesible - Definición y contexto 2014)

El acceso al turismo es un derecho universal garantizado por la Convención de las Naciones Unidas sobre los Derechos de las Personas con discapacidad, tal y como recoge la Red Europea de Turismo Accesible (ENAT). Por determinados factores, el sector de población que demanda turismo accesible es cada vez más numeroso y como consecuencia se han convertido en el segmento que mayor protagonismo ha cobrado en los últimos años en el ámbito turístico tal y como señala Fernández, (2009).

Partiendo de estas premisas, podemos decir que existen diversas razones que justifican el desarrollo de políticas y actuaciones encaminadas a la consecución de un verdadero turismo accesible, tal y como se expone en el Mercado potencial del turismo accesible para el sector turístico de España, de la Secretaría General de Turismo, entre las cuales podemos destacar las siguientes: 
- El turismo es un elemento básico en la vida cotidiana, un derecho en nuestra sociedad. Se debe garantizar por tanto que cualquier persona puede acceder al ejercicio de este derecho independientemente de sus características.

- $\quad$ El turismo es un factor de integración social, que posibilita a las personas alcanzar un determinado nivel de bienestar social.

- $\quad$ La accesibilidad es un elemento básico de calidad de la oferta turística. En este sentido podemos decir que no existe un destino turístico de calidad si este no garantiza la accesibilidad en el mismo, se está convirtiendo por tanto en un elemento indispensable en las demandas del turismo.

- $\quad$ Aumenta la cuota de mercado. La captación de este segmento de la población permite aumentar considerablemente la cuota de mercado actual, contribuyendo a un aumento de los niveles de rentabilidad empresarial.

- Permite la captación del denominado en muchos casos "Multicliente”. Según el manual editado por la Comisión Europea, "Por una Europa Accesible a Turistas con Discapacidades", las cifras sobre el potencial turístico de los ciudadanos europeos con discapacidades no son concluyentes, ya que desde un punto de vista turístico prudente, se calcula que cada viaje realizado por una persona discapacitada atrae a 1,5 acompañantes.

- El desarrollo del turismo accesible favorece la desestacionalización. Esta realidad se basa principalmente en el elevado número de personas con discapacidad y movilidad reducida que no tienen responsabilidades laborales y pueden viajar en cualquier época del año.

- Mejora la imagen de los destinos turísticos y potencia la competitividad. Las diferencias de imagen entre destinos turísticos, en muchas ocasiones, no están en sus productos, sino en el disfrute emocional que se hace de ellos, y en la experiencia personal, en cómo se vive y se disfruta del turismo en un destino determinado.

Asimismo, debemos destacar que la accesibilidad no es un requerimiento únicamente de las personas con discapacidad o diversidad funcional, ya que no son las únicas beneficiarias de la misma; los destinos turísticos que tengan en cuenta estos requisitos, y los entiendan como medidas positivas, evolucionarán en la oferta de sus productos y servicios $\mathrm{y}$, por ende, estarán facilitando la experiencia turística y mejorando la calidad de vida de toda su ciudadanía y visitantes, convirtiéndose en destinos turísticos accesibles de calidad.

\section{Turismo Accesible. Un Derecho Social}

Según la Subdirección General de Calidad e Innovación Turística (2008, p.4)

El concepto de turismo accesible se basa sobre el principio de que el turismo es un derecho social fundamental para todos, a saber, el derecho de la igualdad de oportunidades, a la no discriminación y a la integración social, ya que para las personas con discapacidad, tanto como para todas las demás, las actividades comprendidas bajo las categorías de ocio y turismo son fundamentales para la calidad de vida

La Declaración Universal de los Derechos humanos reconoce a todas las personas el derecho al turismo en las mejores condiciones, y cada vez son más las 
personas con diversidad funcional $\mathrm{y}$ necesidades especiales que reclaman poder participar en la actividad turística y ejercer este derecho. Por ello se están realizando muchas actuaciones encaminadas a favorecer el Turismo Accesible para Todas las personas. Sin embargo, y a pesar del trabajo que se está desarrollando, el cual no podemos obviar, y de los avances que se están consiguiendo en la materia, en la práctica aún existen muchas barreras que impiden o dificultan el acceso de determinadas personas en muchos destinos turísticos. Es importante por tanto destacar que hay muchas personas que presentan diversidad funcional que son potenciales beneficiarias del turismo accesible.

Según la Organización Mundial de la Salud (OMS) una discapacidad "es toda restricción o ausencia (debida a una deficiencia) de la capacidad de realizar una actividad en la forma o dentro del margen que se considera normal para un ser humano"

Atendiendo a los datos de su informe mundial sobre discapacidad, realizado en 2010, más de mil millones de personas, alrededor de un $15 \%$ de la población, viven en todo el mundo con alguna forma de discapacidad; de ellas, casi 200 millones experimentan dificultades considerables en su funcionamiento, y su prevalencia irá en aumento en los próximos años.

Llegados a este punto, debemos también definir el término Diversidad Funcional, el cual ya hemos mencionado con anterioridad y del cual seguiremos citando en adelante. Este término es propuesto por el Foro de Vida Independiente y Diversidad y lo define como "la diferencia de funcionamiento de una persona al realizar las tareas habituales (desplazarse, leer, agarrar, ir al baño, comunicarse, relacionarse, etc.) de manera diferente a la mayoría de la población” (Romañach y Lobato 2005)

Teniendo en cuenta estas consideraciones podemos establecer distintas tipologías de usuarios/as que pueden presentar necesidades especiales a la hora de poder disfrutar plenamente de una experiencia turística, como podemos ver en la siguiente clasificación:

- Diversidad funcional física. En este grupo encontramos a personas usuarias de sillas de ruedas (tanto manuales como motoras) y a personas con movilidad reducida.

- Diversidad funcional sensorial visual. Personas ciegas o con deficiencia visual.

- Diversidad funcional sensorial auditiva, en el que se incluyen las personas ciegas o con deficiencia visual.

- Diversidad funcional intelectual. Personas con capacidades cognitivas restringidas.

- Diversidad funcional orgánica. Aquí encontramos a personas que presentan dificultades respiratorias, o requieren dietas específicas.

Las características que presentan estas personas hacen que puedan encontrar dificultades a la hora de animarse a realizar una experiencia turística, lo cual causa que en muchas ocasiones renuncien a la misma.

Asimismo debemos destacar el hecho de que todas las personas somos diferentes (capacidades, altura, peso, etc.) por lo que tenemos necesidades diferentes. Por ello, a la hora de diseñar servicios y productos se debe tener en cuenta la diversidad humana.

Es prioritario por tanto trabajar para conseguir la participación en la actividad turística de todas estas personas, que presentan capacidades y/o características distintas, 
para favorecer su integración social y mejorar de esta forma su autonomía personal, proporcionando simultáneamente un turismo de calidad y garantizando el ejercicio del derecho al Turismo para todas las personas.

Además es fundamental destacar que estos grupos no son los únicos beneficiarios de la accesibilidad. Los destinos turísticos que trabajen en pro de la misma y lo entiendan como una medida positiva, evolucionarán y mejorarán en la oferta de sus productos y servicios, ofreciendo por tanto una mejor experiencia turística y mejorando la calidad de vida de todos sus ciudadanos/as y visitantes. (Manual sobre Turismo Accesible para Todos: Principios, herramientas y buenas prácticas. Módulo I: Turismo Accesible - definición y contexto, 2014)

\section{Barreras susceptibles de visibilización}

Entre la población potencialmente usuaria de la oferta turística hay colectivos de personas con algún tipo de limitación, discapacidad o diversidad funcional, que continuamente se enfrentan a barreras que les impiden disfrutar de unos determinados servicios, lo que les supone tener que limitar su experiencias de ocio y tiempo libre.

Centrándonos en las personas que presentan algún tipo de discapacidad intelectual, como colectivo objeto de nuestro estudio, cabe señalar que presentan un menor grado de comprensión, orientación y capacidad de toma de decisiones, y en consecuencia, requieren una información sencilla y sistemas de orientación de fácil compresión. Por ello, es necesario visibilizar todos aquellos obstáculos y barreras que dificultan o impiden el disfrute de un turismo de calidad.

Atendiendo a la información proporcionada por el Servicio de Atención a la Discapacidad (SIAD) podemos distinguir entre tres tipos de barreras que afectan a las personas con discapacidad: barreras intrínsecas, ambientales e interactivas.

- Barreras intrínsecas. Vinculadas con los niveles de funcionalidad física, psicológica o cognitiva de cada individuo. Estas barreras pueden estar directamente relacionadas con sus discapacidades específicas, tales como sus problemas de salud o su dependencia física, pero también con otros factores que son secundarios a su diagnóstico médico, tales como sobre-protección familiar o falta de igualdad de oportunidades en la educación.

- Barreras ambientales. Impuestas específicamente por las condiciones físicas o sociales del medio: barreras arquitectónicas, del transporte, ecológicas, de comunicación, o simplemente, de actitud o de rechazo.

- Barreras interactivas. Las relativas a la habilidad requerida para determinadas actividades (dificultad de uso de máquinas o mecanismos de operación o pago). También las relativas a las necesidades de comunicación. Estas se derivan de limitaciones cognitiva o del habla, la audición o la vista. Con independencia de su origen, este tipo de barreras raramente se produce en un solo sentido: la comunicación es un proceso recíproco que requiere la participación activa tanto del que emite el mensaje como del que lo recibe.

Todas estas barreras o limitaciones pueden conducir a las personas que las padecen a situaciones de inseguridad y de aislamiento. Además, si estas barreras las trasladamos al ámbito turístico, la situación se hace más compleja, dificultando y poniendo en riesgo la posibilidad de disfrutar de una experiencia turística de calidad.

Como hemos mencionado anteriormente nos encontramos ante un grupo de personas que tienen diferentes capacidades a la hora de recibir determinada 
información, y de enfrentarse a determinadas situaciones, por tanto es obligatorio pensar en diferentes escenarios y dificultades que pueden encontrarse para trabajar en pro de la eliminación de las mismas.

Entre estas dificultades podemos destacar las siguientes:

- La señalización. Es fundamental una correcta señalización e información en vestíbulos y pasillos, planos, para aquellas personas que se encuentran en un espacio por primera vez.

- Los distintos tiempos de respuesta ante el mismo estímulo son otros de los elementos que hay que tener en cuenta ante la utilización de determinados dispositivos controlados por tiempo, ya que todo el mundo no reacciona ante los mismos con la misma velocidad, como por ejemplo las puertas de un ascensor.

- Otra dificultad es el uso de lenguaje complicado en carteles y señalética.

- Dificultad en la comprensión de la información visual y auditiva.

- Dificultad en la capacidad de memorizar espacios o datos, tanto a largo como a corto plazo.

- Menor capacidad para tomar decisiones o escoger rutas.

- Las distintas capacidades de orientación.

- Las limitaciones que algunas personas pueden presentar en su autonomía personal.

Todas estas barreras hacen que los servicios turísticos no sean accesibles en multitud de ocasiones para este colectivo, provocando por tanto desigualdad en el acceso a los mismos de este segmento de la población, privándoles de un derecho fundamental y provocando por ende una situación de exclusión social.

Es por tanto imprescindible y de rigor analizar e identificar los factores que inciden negativamente en el acceso al turismo de las personas con discapacidad o diversidad funcional intelectual y trabajar activamente en la eliminación de los mismos, tomando las medidas oportunas para crear entornos de calidad, que posibilite un disfrute normalizado de las experiencias turísticas.

Para la eliminación de estas barreras hay que trabajar activamente en las políticas existentes, incidir en la falta de servicios adecuados, y de financiación, y muy importante también insistiendo en la eliminación de prejuicios y actitudes negativas. Todo ello nos lleva a avanzar en materia de accesibilidad turística hacia la consecución del Turismo inclusivo.

\section{Hacia el Turismo inclusivo, Turismo de calidad.}

Llegados a este punto debemos centrarnos en el Turismo inclusivo, el cual supone un paso más en relación al turismo accesible, puesto que este ofrece una serie de servicios que pueden ser disfrutados por personas con capacidades diferentes, pero a su vez son productos que se pueden utilizar por toda la ciudadanía.

El turismo inclusivo, basado en la Atención Integral y Centrada en la Persona, deberá promover las condiciones necesarias para la consecución de mejoras en todos los ámbitos de la calidad de vida y el bienestar de las personas, partiendo del respeto pleno a su dignidad y derechos, de sus intereses y preferencias y contando con su participación efectiva (Rodríguez, 2014). 
Al hablar de turismo inclusivo hablamos por tanto de un turismo que tiene en cuenta la diversidad humana y por ende de un turismo de calidad. El ser diferente o presentar una diversidad funcional no puede ser un impedimento para poder disfrutar de los atractivos que presenta el turismo. Es por tanto fundamental trabajar por la difusión de la importancia del mismo, de ocuparse y preocuparse de manera coordinada en la consecución de este turismo de calidad para todas las personas, ya que el turismo proporciona múltiples vivencias, permite descansar de la rutina diaria, conocer otras realidades y culturas y la interrelación con otras personas, lo cual reporta un gran número de beneficios personales.

Teniendo en cuenta que el Turismo inclusivo favorece la participación social en igualdad de oportunidades para todas las personas en el caso de las personas que presentan algún tipo de diversidad funcional ejerce un papel un papel fundamental ya que permite el disfrute de esta experiencia de una manera plena y satisfactoria.

El poder disfrutar del turismo de una manera inclusiva ofrece una serie de beneficios no sólo para las personas que presentan algún tipo de diversidad funcional, sino también para sus familiares. Entre ellos cabe destacar:

- La posibilidad de disfrutar de experiencias turísticas de calidad.

- Favorece la participación en el ocio y la cultura.

- Mejora la calidad de la experiencia del viaje.

- Facilita la inclusión social y favorece la responsabilidad social.

- Las mejoras incorporadas en el diseño para todas las personas aumentan la calidad de vida, al requerir un menor esfuerzo físico o intelectual en el desarrollo de la experiencia turística.

- Favorece la autoconfianza y la dignidad.

En el caso de las personas con la discapacidad intelectual su implicación en las situaciones de la vida incluyendo el ocio y las actividades recreativas, está asociada a la mejora del bienestar emocional, de las relaciones sociales y al desarrollo de sus competencias. (Badia y Longo, 2009)

Por tanto, es fundamental para el disfrute de la actividad turística asegurar que la misma se va a poder llevar a cabo con las garantías necesarias de principio a fin. La experiencia turística se inicia en el lugar de origen, antes de comenzar nuestro viaje con la búsqueda de información sobre el destino, medios de transporte, etc., continúa con el desarrollo de una serie de actividades en el lugar de destino elegido, como pueden ser el alojamiento, la restauración o visitas entre otros, y finaliza cuando se regresa al lugar de origen.

En este sentido es importante resaltar que todas las actividades que se desarrollan en esta experiencia turística son elementos entendidos como una cadena compuesta por muchos eslabones, y donde se deben implicar todos los profesionales que interactúen en este proceso, es muy importante destacar que es fundamental garantizar que cada uno de estos elementos son accesibles, ya que la no accesibilidad de uno de ellos puede condicionar la accesibilidad de todo el conjunto. (Manual sobre Turismo Accesible para Todos, Módulo II, Cadena de accesibilidad y recomendaciones ,2014) 
Atendiendo a las recomendaciones del citado Manual, es fundamental poner en valor estos elementos para garantizar el uso y disfrute de un viaje para cualquier persona y para ello debemos tener las siguientes consideraciones en cada uno de ellos:

\section{Planificación e información}

Este paso es fundamental antes de emprender cualquier viaje. En el caso del turismo accesible es prioritario facilitar información sobre lugares y centros turísticos accesibles. Para ello se utilizan normalmente diversos canales, entre ellos destacamos Internet como el más utilizado y también los turoperadores y las agencias de viajes.

Es básico por tanto la existencia de páginas web accesibles, guías de accesibilidad turística fiables e informadores turísticos con formación en materia de turismo accesible

\section{Transporte de salida}

El transporte en el punto de salida es fundamental en un viaje, ya que de nada sirve la existencia de un destino accesible si no podemos llegar a él. Es fundamental destacar que los terminales de los pasajeros sean accesibles y cuenten con la señalización adecuada y que se puedan acceder a todos los servicios existentes. En el caso de los vehículos es necesario que respondan a características como, asientos para personas con discapacidad, espacios habilitados para usuarios en silla de ruedas con sistema de anclaje y aseos accesibles.

Entorno

Una vez llegados al destino es fundamental poder interactuar en él. Para ello se debe hacer previamente una clasificación de los mismos: entornos urbanos, entornos rurales y naturales, entornos marítimos y entornos históricos y patrimoniales. Cada uno ellos tienen unas características particulares que requieren distintas consideraciones:

- Entorno urbano. Es fundamental que se pueda realizar recorridos sobre pavimentos antideslizantes y uniformes, con vados rebajados y señalización adecuada.

- Entorno rural/ natural. Es muy importante garantizar la continuidad y para ello el itinerario deberá contar con suelo firme, estable y continuo, espacio para la circulación libre de obstáculos, zonas de cruce y accesos e iluminación y señalización adecuada.

- Entornos marítimos. Son uno de los principales atractivos turísticos por ello es importante su accesibilidad para todas las personas. Entre las necesidades a satisfacer destacamos el poder circular en las zonas de playa y poder disfrutar de los servicios ofrecidos, así como el acceso hasta el mar, el baño y la salida más segura.

- Entornos históricos y patrimoniales son quizás los más complicados a la hora de convertirlos en accesibles por su propia naturaleza, pero siempre se pueden llevar a cabo medidas que puedan aumentar su accesibilidad Entre estas medidas podemos destacar: proporcionar más espacio peatonal, no estrangular el tráfico rodado ni las posibilidades de estacionamiento, ordenar la ubicación del mobiliario urbano, y evitar itinerarios que acusen fatiga.

Transporte local 
El transporte local se constituye como un elemento clave para posibilitar el desplazamiento en destino.

\section{Alojamiento}

Es fundamental garantizar a la clientela comodidad durante su estancia. Para ello, muy importantes son los siguientes elementos:

- $\quad$ El recorrido hasta el hotel, los accesos al mismo y el entorno, así como en el interior del mismo, (pasillos, ascensores, etc.), deben de poder hacerse en condiciones de seguridad.

- $\quad$ La recepción y el vestíbulo deben de ser amplios y con un diseño adecuado.

- Las habitaciones y el aseo deben contar con adaptaciones reales probadas como el ancho de las puertas, el diseño de los muebles, la altura de las cosas o los planes de evacuación.

- Los espacios comunes como restaurantes, gimnasios, piscinas, etc., también deben de ser accesibles.

No sólo hay que tener en cuenta el acceso al establecimiento y todos los espacios y servicios comunes, sino que además es de suma importancia que el personal de atención al cliente tenga una formación adecuada sobre todos los elementos de accesibilidad del establecimiento.

Restauración, compras, etc

Estos puntos de la actividad turística constituyen un gran atractivo y un incentivo más en el destino turístico. Es por ello muy importante incidir también en esta área seleccionando y adaptando los establecimientos que se consideren necesarios, así como promocionar todos los establecimientos accesibles en el Plan Turístico del lugar

Actividades de ocio

En todas estas ofertas debemos de resaltar un mismo hecho y es que en las actividades de ocio (visitas a museos, teatros, deportes, etc...) es fundamental no sólo el acceso físico, sino también el disfrute de los contenidos.

\section{Excursiones}

Los puntos más importantes a destacar en relación a las excursiones son:

- La creación y promoción de productos específicos

- La coordinación con agencias de viajes para facilitar la adaptación de determinadas excursiones

- $\quad$ Promover y garantizar la existencia de recursos accesibles en el destino

Servicios médicos y de apoyo

Cualquier turista considera requisito imprescindible tener la seguridad de que ante cualquier posible necesidad médica o sanitaria, la misma se va a poder cubrir en el sitio de destino. Es fundamental por tanto garantizar la existencia de un hospital cercano y de equipos de auxilio para situaciones de emergencia; asistencia; farmacias cercanas y ortopedias que ofrezcan la posibilidad de alquilar y/o reparar elementos de apoyo, en caso de necesidad.

Transporte de salida 
llegada

Se debe tener en cuenta las mismas consideraciones que en el transporte de

\section{Experiencia final}

Una vez finalizada la experiencia turística también es muy importante la existencia de sistemas eficaces de recogida de información, (realización de sugerencias, quejas, etc.), que puedan servir a otros posibles usuarios y también para mejorar la accesibilidad de determinados servicios

Asimismo, y siguiendo con las recomendaciones de este manual cabe destacar que para garantizar el cumplimiento de la cadena de accesibilidad, son necesarios, algunos elementos que van más allá de las infraestructuras y entornos construidos, a saber:

- $\quad$ La información sobre accesibilidad debe ser una parte integral de la información turística general.

- La tecnología debe poder ser utilizada por todas las personas que la usen en la más amplia gama de situaciones sin diseño especial.

- Todo el personal debe tener las competencias necesarias para dar cabida a las personas con discapacidad.

Todos estos elementos son esenciales en la cadena de accesibilidad en turismo, puesto que nada sirve, por ejemplo, alojarse en un hotel totalmente accesible si luego no se puede disfrutar del entorno o en el regreso no se puede compartir la experiencia con otras personas bien sea de modo físico o a través de las redes sociales virtuales. Es fundamental por tanto poner en valor la cadena del turismo accesible, para garantizar una experiencia turística de calidad.

\section{Discusión y conclusiones}

Se bien se ha avanzado mucho en materia de accesibilidad, en el ámbito turístico aún queda mucho camino por recorrer. La apuesta va dirigida hacia un turismo inclusivo de calidad que permita a todas las personas, más allá de sus capacidades y características personales, poder disfrutar de la experiencia como derecho social que es.

No obstante como ya comentáramos aún las ofertas turísticas inclusivas son escasas y por ende, las personas con algún tipo de discapacidad en general y con discapacidad intelectual en particular, todavía se encuentran con múltiples dificultades de acceso, lo cual obstaculiza el ejercicio de este derecho y les propicia a la vez un mayor aislamiento social.

Es constatable que se ha trabajado mucho durante los últimos tiempos para la consecución de la accesibilidad física, no obstante, las medidas adoptadas para la accesibilidad de personas con discapacidad cognitiva y/o sensorial han sido bastante más escasas, por ello es necesario potenciar e intensificar estrategias que favorezcan dicha accesibilidad.

Asimismo cabe destacar el avance en el acceso al turismo de ciertos colectivos mediante la creación de determinados paquetes turísticos sectoriales enfocados a un determinado tipo de discapacidad, de modo que les permite disfrutar de experiencias turísticas concretas, pero reiteramos que las líneas de actuación futuras deben ir 
encaminadas a la consecución de entornos que permitan disfrutar a todas las personas por igual de una determinada experiencia turística, independientemente de sus características consiguiendo de esta manera un real y efectivo turismo inclusivo.

Es primordial por tanto continuar en la labor de propiciar una mejora en las ofertas turísticas, que posibiliten el ejercicio de este derecho a todas las personas por igual. Se trata de trabajar de manera conjunta y multidisciplinar en todos los eslabones que conforman la cadena de accesibilidad, para poder ofertar paquetes turísticos de calidad.

Asimismo otro punto clave de este tema es la concienciación y formación en la materia de todos los agentes implicados y de la sociedad en general. Se debe por tanto potenciar y favorecer la inclusión social y la igualdad de oportunidades para posibilitar que todas las personas puedan vivir en cualquier ámbito de la sociedad de igual a igual, incluyendo por supuesto el acceso al ocio, a la cultura y al turismo.

En definitiva, desde todos los ámbitos, empresarial, político y social, se deben aunar fuerzas y trabajar hacia la consecución de destinos turísticos inclusivos que tengan en cuenta las necesidades de las personas con independencia de sus capacidades funcionales. Se deben crear productos universales mediante los cuales poder disfrutar conjuntamente de las experiencias turísticas, puesto que la creación de productos específicos para las personas que presentan alguna necesidad especial, si bien posibilitan el poder realizar una determinada actividad, favorecen la exclusión al no poder realizarla con y en las mismas condiciones que el resto.

\section{Referencias}

Álvarez, J. (2015). Smart Human City. Hacia una ciudad inteligente para todas las personas. I Congreso Ciudades Inteligentes. Madrid.

Badia, M y Longo, E.(2009) "El ocio en las personas con discapacidad intelectual: participación y calidad de vida a través de las actividades de ocio" Revista Española sobre Discapacidad Intelectual, 40 (3), 231.

De La Fuente, Y.M.; Martín, M.C y Hernández, J. (2016). "El nuevo paradigma de la accesibilidad. Rompiendo Barreras Invisibles". En Políticas e Intervenciones Sociales ante los procesos de Vulnerabilidad y Exclusión de Personas y Territorio. Un análisis comparado México-España. Madrid: Editoral Dikison.

EIDD Desing for All Europe (2004). The EIDD Stockholm Declaration Adopted on 9 May 2004, at the Annual General Meeting of the European Institute for Design and Disability in Stockholm. Recuperado de http://www.designforalleurope.org/Design-for-All/EIDDDocuments/Stockholm-Declaration

Fernández, M. T. (2009) "Turismo Accesible: la importancia de la Accesibilidad para el sector turístico”. Entelequia. Revista Interdisciplinar, 9.

Garza, M.C. (2016), Salud y Accesibilidad elementos fundamentales en la igualdad de oportunidades y calidad de vida de las personas con discapacidad. MAW2015.Madrid Accesibility week. Turismo, Tecnología y Accesibilidad. Universidad Internacional de Andalucía. Publicaciones.unia.es 
García, C. (2016), Propuesta de creación departamento de investigación y desarrollo de la accesibilidad y la calidad en el conjunto monumental de la Alhambra y el Generalife. MAW-2015.Madrid Accesibility week. Turismo, Tecnología y Accesibilidad. Universidad Internacional de Andalucía. Publicaciones.unia.es

Guirao, JA (2015). Utilidad y tipos de revisión de literatura. Ene. Revista de enfermería. Recuperado de http://eneenfermeria.org/ojs/index.php/ENE/article/view/495/guirao doi: https://doi.org/10.4321/S1988-348X2015000200002

Icart Isern, M.T. y Canela Soler, J. (1994). El artículo de revisión. Enfermería Clínica, $4,4,180.4$

Ministerio de Sanidad, Política social e Igualdad. Estrategia española sobre discapacidad 2012-20120. Recuperado de: http://www.msssi.gob.es/ssi/discapacidad/docs/estrategia_espanola_discapacida d_2012_2020.pdf

Muñoz, Ma . D. (2014), Módulo los Servicios de la ciudad, Asignatura" Turismo para todos". Tema Turismo Inclusivo .Máster en Accesibilidad para Smart City. Universidad de Jaén.

Organización Mundial de la Salud, (2010). Informe Mundial sobre la Discapacidad. Recuperado de: http://www.who.int/disabilities/world_report/2011/es/

Organización Mundial del Turismo, (2014). Manual sobre Turismo Accesible para Todos: Principios, herramientas y buenas prácticas - Módulo I: Turismo Accesible - definición y contexto. Recuperado de: http://dtxtq4w60xqpw.cloudfront.net/sites/all/files/docpdf/manualturismoaccem oduloipruebadia14final1 revisadov2.pdf

Organización Mundial del Turismo, (2014). Manual sobre Turismo Accesible para Todos: Principios, herramientas y buenas prácticas - Módulo II: Cadena de Accesibilidad. Recuperado de: http://www.fundaciononce.es/sites/default/files/manual_sobre_turismo_accesibl e._modulo_ii_cadena_de_accesibilidad_y_recomendaciones_0.pdf

Ramos, M.H; Ramos, M.F. y Romero, E. (2003). Cómo escribir un artículo de revisión. Revista de postgrado de la VIa Catedra de Medicina. Recuperado de http://med.unne.edu.ar/revista/revista126/como_esc_articulo.htm.

Red Europea para el Turismo Accesible, EANAT. Trabajando juntos para hacer el turismo en Europa accesible a todos. (2006).Recuperado de: http://www.accessibletourism.org/resources/enat-a4_spanish.pdf

Rodríguez, P. (2014), La atención integral y centrada en la persona Recuperado de: http://www.fundacionpilares.org/docs/AICPweb.pdf

Romañach, J, Lobato, M, (2005), Diversidad funcional, nuevo término para la lucha por la dignidad en la diversidad del ser humano. Foro de vida independiente. Recuperado de: http://www.forovidaindependiente.org/files/documentos/pdf/diversidad_funcion al.pdf

Secretaría de Turismo, España (2008), El mercado potencial del turismo accesible para el sector turístico español. Recuperado de: 
http://planaccesibilidadturistica.es/UserFiles/publicaciones/ficheros/Mercado_Po tencial_Turismo_Accesible.pdf

Servicio de Información Sobre Discapacidad. Ministerio de Sanidad, Servicios Sociales e Igualdad. Capítulo 1. Dimensiones de la Accesibilidad. Conceptos y elementos de base para el diagnóstico. Recuperado de: http://sid.usal.es/idocs/F8/8.15999/Parte\%20I/cap_1.htm

Fecha de recepción: 11/10/2017

Fecha de revisión: $31 / 10 / 2017$

Fecha de aceptación: 10/03/2018 
